\title{
An Equation for Numerical Computation of Self-Diffusion Coefficient in Salt Baths
}

\author{
By Kazutaka Kawamura* and Tsutomu Ando**
}

\begin{abstract}
The reasonable choice of an equation among the equations for the determination of self-diffusion coefficients from the data obtained by the capillary reservoir method is elucidated by developing an alternative form of the well-known equation. The obtained alternative form is proved to be identical theoretically with the well-defined equation. It is also shown by using the alternative form that the equation

$$
C_{a}=C_{0}\left(1-\frac{2}{l} \sqrt{\frac{D t}{\pi}}\right)
$$

can be used at a lower value of $\sqrt{D_{t}} / l$ than $0.5_{0}$ and that the equation

$$
C_{a}=\frac{8 C_{0}}{\pi^{2}} e^{-\frac{D \pi^{2} t}{4 l^{2}}}
$$

can be valid at a higher value of $\sqrt{\bar{D} t} / l$ than $0.4_{2}$.

The calculated value of self-diffusion coefficients of silver ion in pure molten sodium nitrate by an appropriate equation provide the validity of using the chosen equations at a given value of $\sqrt{D t} / l$. The obtained diffusion coefficient agrees well with the chronopotentiometric and polarographic diffusion coefficients of silver ion in pure molten sodium nitrate.

(Received September 8, 1971)
\end{abstract}

\section{Introduction}

It has been well known that molten salts have extensively been employed for the heat treatment of ferrous and non-ferrous alloys. In particular, salt baths of alkali metal nitrates have been used over a wide temperature range from 180 to $600^{\circ} \mathrm{C}^{(1)}$. However, some reactions between metals and salt baths, such as a corrosion, are encountered, and the diffusion coefficients of metallic ions frequently play an important role in such a reaction.

Diffusion coefficients of various ions in molten alkali metal nitrate melts have been determined by the electrochemical method ${ }^{(2) \sim(7)}$, the diaphragm cell method ${ }^{(8)(9)}$, the optical method ${ }^{(10)(11)}$, the radioactive isotope (tracer) method ${ }^{(12) \sim(17)}$ and the pulsed NMR method ${ }^{(18)}$. The self-diffusion coefficients, however, have mainly been obtained by the radioactive isotope method. Although many reports have dealt with self-diffusion coefficients of dilute metallic ions in pure molten alkali metal nitrates, a little has been known about self-diffusion

* National Research Institute for Metals, Meguro-ku, Tokyo. Present address : Research Laboratory of Nuclear Reactor, Tokyo Institute of Technology, Meguro-ku, Tokyo, Japan.

** National Research Institute for Metals, Meguro-ku, Tokyo, Japan.

(1) K, Kawamura : Kagaku no Ryoiki, 23 (1969), 494.

(2) K. Kawamura : AEC Report TID-20866, (1963), p. 13

(3) K. Kawamura : Electrochimica Acta, 12(1967), 1233.

(4) K. Kawamura : Denki Kagaku, 38 (1970), 12.

(5) C. E. Thalmayer, S. Bruckenstein and D. M. Gruen : J. Inorg. Nucl. Chem., 26 (1964), 347.

(6) J. E. L. Bowcott and B. A. Plunkett : Electrochimica Acta, 14 (1969), 363.

(7) S. Sternberg and C. Herdlicka : Revue Roumaine de Chimie, 14 (1969), 991.

(8) R.W. Laity and M. P. Miller : J. Phys. Chem., 68 (1964), 2145.

(9) C. A. Sjəblom : Z. Naturforsch., 20 a (1965), 1572.

(10) S. E. Gustaffson, L. E. Wallin and T.E. G. Arvidsson : Z. Naturforsch., 23 a (1968), 1261.

Trans. J I M coefficients of metallic ions in the whole range of binary alkali metal nitrates ${ }^{(19) \sim(22)}$. The present authors carried out a series of studies in order to clarify experimentally the variation of diffusion coefficients of metallic ions, particularly of silver ion, in various binary silver nitrate-alkali metal nitrates and to explain it in terms of physical parameters systematically.

The purpose of the present paper is to elucidate how selection of an equation among various equations, by which to calculate the diffusion coefficient from the data obtained by the capillary reservoir method, is useful for the simplification of numerical computation under a given experimental condition; how the selected equation relates to other equations by using the newly derived equation; and how the diffusion coefficients of silver ion in pure sodium nitrate, calculated from the equation selected on the basis of the data obtained under various experimental conditions, are in good agreement with those calculated from other equations.

(11) T. E. G. Arvidsson, S. A. Afsenius and S. E. Gustaffson : J. Chem. Phys., 53 (1970), 2621.

(12) G. J. Janz: Molten Salt Handbook, Academic Press, (1967), p. 351.

(13) A.S. Dworkin, R.B. Escue and E.R. Van Artsdalen : J. Phys. Chem., 64 (1960), 872.

(14) E. P. Honig and J.A. A. Ketelaar : Trans. Faraday Soc., 62 (1966), 190.

(15) C. A. Angell and J. W. Tomlinson : Discussions Faraday Soc., 32 (1961), 237.

(16) J. A. A. Ketelaar and E. P. Honig : J. Phys. Chem., 63 (1964), 1596.

(17) H. J. Arniker : Compt. rend., 244 (1957), 2241.

(18) K. Kawamura and K. Nakamura : to be published.

(19) S. Forcheri and V. Wagner : Z. Naturforsch., 22 a (1967), 1171.

(20) F. Lantelme and M. Chemla : Bull. Soc. Chim. France, (1963), 969.

(21) F. Lantelme and M. Chemla : Electrochimica Acta, 11 (1966), 1023.

(22) J. A. A. Ketelaar and J. C. Th. Kwak : Trans. Faraday Soc., 65 (1969), 139. 


\section{Selection of Equation}

The capillary reservoir method can be divided into two : (a) The radioactive isotope in capillary diffuses in the reservoir containing the same salt unlabeled. After the lapse of a certain time, the capillary is removed. Its contents are analyzed for the total quantity of the remaining labeled ion. The self-diffusion coefficient is calculated from the ratio of initial to final concentration. (b) In contrast with (a), the radioactive isotope in the reservoir diffuses in the capillary containing the same salt unlabeled. In addition, there are two cases in each method: (a) The so-called diffusion length is larger than the capillary length. (b) The so-called diffusion length is smaller than the capillary length. The proposed equations for all cases are summarized in Table 1.

Let us consider the following two equations for the capillary filled with radioactive isotope : can be written when the term, $\frac{8 C_{0}}{\pi^{2}} \sum_{n=1}^{\infty} \frac{1}{(2 n+1)^{2}} e^{-\frac{D(2 n+1) 2 \pi 2 t}{4 l 2}}$, becomes negligibly small.

Next, we try to demonstrate the experimental conditions under which a simple form, such as eq. (2) and (4), can be used to calculate the diffusion coefficients and to explain such conditions in terms of $\sqrt{D t} / l$ instead of the so-called diffusion length. If the difference between eq.(1) and eq. (2) is found to be small within the experimental error under a given $\sqrt{\overline{D t} / l}$, eq. (2) may be used for the determination of diffusion coefficient. Instead of the direct numerical computation of (eq.(1) eq. (2)), however, such a computation can be carried out easily by using an alternative form of eq.(1), i.e., eq. (3). The relation between ((eq. (3) - eq. (2))/eq. (2)) $\times 100$ and $\sqrt{D t} / l$ is shown in Fig. 1. It is found from this figure that eq.(1), i. e., eq.(3), can be replaced by eq.(2)

Table 1 Various equations for determination of diffusion coefficients by the capillary reservoir method.

\begin{tabular}{c|c|c}
\hline \hline & R. I. in reservoir & R. I. in capillary \\
\hline \multirow{2}{*}{$<$ Diffusion length } & $C_{a}=C_{0}-\frac{8 C_{0}}{\pi^{2}} \sum_{n=0}^{\infty} \frac{1}{(2 n+1)^{2}} e^{-\frac{D(2 n+1) 2 \pi 2 t}{4 l 2}}$ & $C_{a}=\frac{8 C_{0}}{\pi^{2}} \sum_{n=0}^{\infty} \frac{1}{(2 n+1)^{2}} e^{-\frac{D(2 n+1) \pi^{2 t}}{4 l 2}}$ \\
\hline$l>$ Diffusion length & $C_{a}=\frac{2 C_{0}}{l} \sqrt{\frac{D t}{\pi}}$ & $\left.C_{a}=C_{0} 1-\frac{2}{l} \sqrt{\frac{D t}{\pi}}\right)$ \\
\hline \hline
\end{tabular}

$$
\begin{aligned}
& C_{a}=\frac{8 C_{0}}{\pi^{2}} \sum_{n=0}^{\infty} \frac{1}{(2 n+1)^{2}} e^{-\frac{D(2 n+1) 2_{\pi t 2}}{4 l 2}}, \\
& C_{a}=C_{0}\left(1-\frac{2}{l} \sqrt{\frac{D t}{\pi}}\right),
\end{aligned}
$$

where $C_{a}$ is the average concentration in the capillary after the diffusion, $C_{0}$ is the initial concentration in the capillary, $D$ is the diffusion coefficient $\left(\mathrm{cm}^{2} / \mathrm{sec}\right), l$ is the length of the capillary (cm), $t$ is the time lapse (sec) and $n$ is the number of terms. Since such well-known two equations are dissimilar to each other, some experimenters are confused in calculating the diffusion coefficients from the obtained data. It is, thus, very helpful to derive a new equation which has the same form as eq. (2) and is completely equivalent to eq. (1). As shown in Appendix 1 and Appendix 2, it is seen that a following equation can fulfil the above conditions.

$C_{a}=C_{0}\left(1-\frac{2}{l} \sqrt{\frac{\overline{D t}}{\pi}}\left(1+2 \sqrt{\pi} \sum_{n=1}^{\infty}(-1)^{n} i \operatorname{erfc} \frac{n l}{\sqrt{D t}}\right)\right)$.

From eq. (3) it is easily seen that eq. (2) is obtained when the right side term of eq. (3), i.e.,

$\frac{4 C_{0}}{l} \sqrt{D t} \sum_{n=1}^{\infty}(-1)^{n} i \operatorname{erfc} \frac{n l}{\sqrt{D t}}$, becomes negligibly small.

On the other hand, the equation

$$
C_{a}=\frac{8 C_{0}}{\pi^{2}} e^{-\frac{\mathrm{D}_{\pi} 2 t}{4 / 2}}
$$

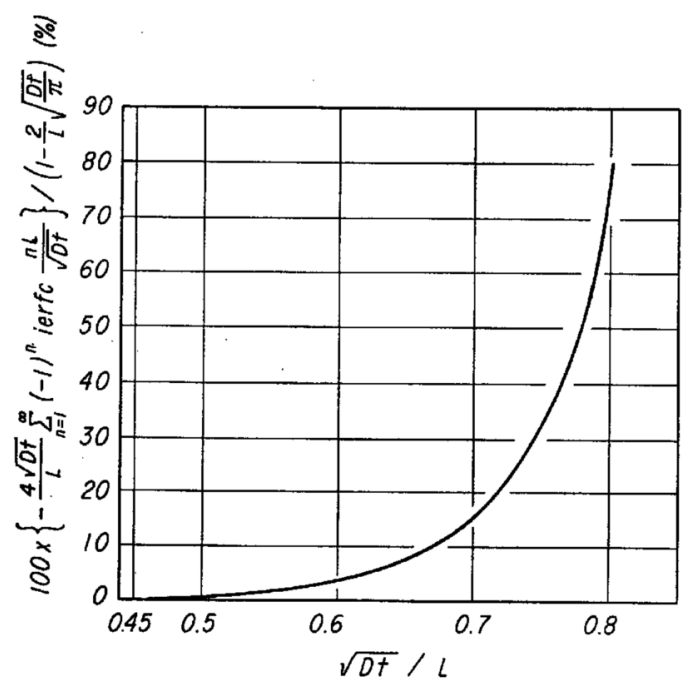

Fig. 1 Per cent difference between eq. (3) and eq. (2).

within the accuracy of $0.5 \% *$ of value of $C_{a} / C_{0}$ when the value of $\sqrt{D t} / l$ is less than $0.5_{0}$. If the value of $\sqrt{D t} / l$ is larger than $0.5_{0}$, the term of the right side of eq. (3) has to be added. As shown in Fig. 2, it is found that the number of term to be added, $n$, is dependent on the value of $\sqrt{\overline{D t} / l}$; for example, the $n$ is two at the value between $1.0_{0}$ and $14_{6}$. As for eq. (1) the same relation as mentioned above is also shown in Fig. 2. As shown in this figure, the convergency of the alternative form, eq. (3), is a contrast to that of eq. (1) and eq.

* This accuracy corresponds to the experimental accuracy. 


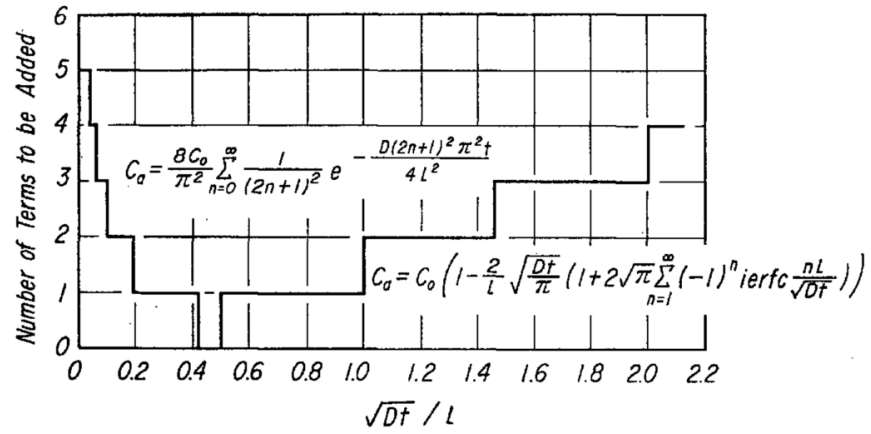

Fig. 2 The relation between the number of terms to be added and $\sqrt{\bar{D} t} / l$.

(1) can be replaced by eq. (4) at the higher value of $\sqrt{D t} / l$ than $0.4_{2}$.

\section{Results and Discussion}

The experiment was achieved by using a capillary* about $5 \mathrm{~cm}$ in length, in which the pure sodium nitrate with small amounts of silver ion isotope was filled, under the various diffusion time from 10 to $120 \mathrm{hr}$ at $350^{\circ} \mathrm{C}$ and $20 \sim 40 \mathrm{rpm}$. The numerically or graphically calculated diffusion coefficients by using the various equations, i.e., eqs.(1), (2), (3) and (4), are summarized in Table 2 together with the experimental values. The values from eq. (2) also agree with that from eq.(1) at $\sqrt{D t} / l$ of between $0.1_{7}$ and $0.4_{2}$ (giving the diffusion time of between 10 and $49 \mathrm{hr}$ ). As can be predicted from Fig. 2, these results provide the justification of the choice of equation.

The obtained diffusion coefficients show the tendency to decrease with diffusion time until $40 \mathrm{hr}$ and the constant values over $40 \mathrm{hr}$. Although the reason for the increase of diffusion coefficient at short diffusion time is not clear, it might be caused by the disturbance at the open end of the capillary when the capillary is immersed into the melt of the reservoir. The effect of such disturbance on the diffusion coefficient may decrease gradually with the lapse of diffusion time.

It is to be noted finally that a chronopotentiometric and polarographic studies of silver ion in pure molten sodium nitrate carried out in this laboratory ${ }^{(3)}$ appears to confirm the validity of the value of obtained diffusion coefficient of silver ion.

\section{Conclusion}

In order to find out an equation among the equations for the determination of diff usion coefficient under a given experimental condition of the capillary (filled with the radioactive isotope) reservoir method, the alternative form

Table 2 Self-diffusion coefficients of silver ion in pure molten sodium nitrate.

\begin{tabular}{|c|c|c|c|c|c|c|c|c|c|}
\hline \multirow{2}{*}{$t(\mathrm{hr})$} & \multirow{2}{*}{$\left(C_{0}-C_{a}\right) / C_{0}$} & \multirow{2}{*}{$l(\mathrm{~cm})$} & \multirow{2}{*}{$\sqrt{D t} / l$} & \multicolumn{2}{|c|}{$\begin{array}{l}D \text { calculated from } \\
\text { eq. (3) or eq. ( } 2)\end{array}$} & \multicolumn{4}{|c|}{$\begin{array}{l}D \text { calculated from } \\
\text { eq. (1) or eq. (4) }\end{array}$} \\
\hline & & & & $D \times 10^{-5}\left(\mathrm{~cm}^{2} / \mathrm{sec}\right)$ & $n$ & $D \times 10^{-5}\left(\mathrm{~cm}^{2} / \mathrm{sec}\right)$ & & $n$ & \\
\hline $\begin{array}{c}120 \\
120 \\
120 \\
120 \\
92.6 \\
92.6 \\
92.6 \\
92.6 \\
70 \\
70 \\
70 \\
49 \\
49 \\
49 \\
41.3 \\
41.3 \\
41.3 \\
20 \\
20 \\
20 \\
9.9 \\
\mathbf{9 . 9} \\
\mathbf{9 . 9} \\
\mathbf{9 . 9} \\
\mathbf{9 . 9}\end{array}$ & $\begin{array}{l}0.66_{8} \\
0.66_{9} \\
0.68_{5} \\
0.65_{8} \\
0.60_{9} \\
0.60_{0} \\
0.60_{4} \\
0.59_{7} \\
0.53_{3} \\
0.53_{4} \\
0.53_{5} \\
0.43_{7} \\
0.44_{2} \\
0.43_{8} \\
0.44_{7} \\
0.44_{5} \\
0.41_{7} \\
0.29_{2} \\
0.29_{5} \\
0.29_{0} \\
0.22_{3} \\
0.19_{6} \\
0.20_{4} \\
0.21_{9} \\
0.19_{6}\end{array}$ & $\begin{array}{l}5.44 \\
5.36 \\
5.23 \\
5.53 \\
5.26 \\
5.34 \\
5.28 \\
5.28 \\
5.28 \\
5.22 \\
5.20 \\
5.42 \\
5.35 \\
5.43 \\
4.78 \\
4.76 \\
5.21 \\
5.36 \\
5.34 \\
5.42 \\
5.15 \\
5.32 \\
5.36 \\
5.28 \\
5.41\end{array}$ & $\begin{array}{l}0.6_{0} \\
0.6_{0} \\
0.6_{2} \\
0.5_{9} \\
0.5_{4} \\
0.5_{4} \\
0.5_{4} \\
0.5_{3} \\
0.4_{7} \\
0.4_{7} \\
0.4_{8} \\
0.3_{9} \\
0.3_{9} \\
0.3_{8} \\
0.4_{0} \\
0.4_{0} \\
0.3_{7} \\
0.2_{7} \\
0.2_{6} \\
0.2_{6} \\
0.1_{9} \\
0.1_{7} \\
0.1_{8} \\
0.2_{0} \\
0.1_{7}\end{array}$ & $\begin{array}{l}2.4_{8} \\
2.4_{2} \\
2.4_{3} \\
2.4_{7} \\
2.4_{5} \\
2.4_{5} \\
2.4_{3} \\
2.3_{7} \\
2.4_{7} \\
2.4_{2} \\
2.4_{2} \\
2.5_{0} \\
2.4_{9} \\
2.5_{2} \\
2.4_{2} \\
2.4_{7} \\
2.5_{0} \\
2.6_{8} \\
2.6_{9} \\
2.6_{9} \\
2.9_{1} \\
2.3_{9} \\
2.6_{3} \\
2.9_{4} \\
2.4_{7}\end{array}$ & $\begin{array}{ll}0, & 1 \\
0, & 1 \\
0, & 1 \\
0, & 1 \\
0, & 1 \\
0, & 1 \\
0, & 1 \\
0, & 1 \\
0 & \\
0 & \\
0 & \\
0 & \\
0 & \\
0 \\
0 \\
0 \\
0 \\
0 \\
0 \\
0 \\
0 \\
0 \\
0 \\
0 \\
0\end{array}$ & $\begin{array}{l}2.4_{8} \\
2.4_{1} \\
2.4_{2} \\
2.4_{7} \\
2.4_{5} \\
2.4_{5} \\
2.4_{3} \\
2.3_{7} \\
2.4_{7} \\
2.4_{3} \\
2.4_{2} \\
2.5_{0} \\
2.4_{9} \\
2.5_{2} \\
2.4_{1} \\
2.4_{8} \\
2.5_{0} \\
2.6_{8} \\
2.7_{0} \\
2.6_{9} \\
2.9_{1} \\
2.3_{9} \\
2.6_{3} \\
2.9_{4} \\
2.4_{8}\end{array}$ & $\begin{array}{l}0 \\
0 \\
0 \\
0 \\
0 \\
0 \\
0 \\
0 \\
0 \\
0 \\
0 \\
0 \\
0, \\
0, \\
0, \\
0, \\
0, \\
0, \\
0, \\
0, \\
0, \\
0, \\
0, \\
0, \\
0, \\
0,\end{array}$ & $\begin{array}{l}1 \\
1 \\
1 \\
1 \\
1 \\
1 \\
1 \\
1 \\
1 \\
1, \\
1, \\
1, \\
1, \\
1,\end{array}$ & $\begin{array}{l}2 \\
2 \\
2 \\
2 \\
2\end{array}$ \\
\hline
\end{tabular}

values of diffusion coefficients listed in the table show that the calculated values form eq. (4) agree with those from eq. (3) at $\sqrt{\overline{D t} / l}$ of between $0.5_{3}$ and $0.6_{0}$ (giving the diffusion time of between 90 and $120 \mathrm{hr}$ ), the values from eq. (2) agree with that from eq. (4) at $\sqrt{D t / l}$ of $0.4_{7} \sim 0.4_{8}$ (giving the diffusion time of $70 \mathrm{hr}$ ) and the

* Taking $D=2 \times 10^{-5} \mathrm{~cm}^{2} / \mathrm{sec}$ and $l=5 \mathrm{~cm}$, one obtains the diffusion time of about $22 \mathrm{hr}$, at which the so-called diffusion length becomes equal to the length of capillary.
$C_{a}=C_{0}\left(1-\frac{2}{l} \sqrt{\frac{D t}{\pi}}\left(1+2 \sqrt{\pi} \sum_{n=1}^{\infty}(-1)^{n} i \operatorname{erfc} \frac{n l}{\sqrt{D t}}\right)\right)$

of well-known equation

$$
C_{a}=\frac{8 C_{0}}{\pi^{2}} \sum_{n=0}^{\infty} \frac{1}{(2 n+1)^{2}} e^{-\frac{D(2 n+1) 2 \pi 2 t}{4 l 2}}
$$

is elucidated. It is also proved that the former equation is completely identical with the latter theoretically. 
While the former equation converges at a low value of $\sqrt{D t} / l$, the latter equation converges at a high value of $\sqrt{D t / l}$. Thus, the equation

$$
C_{a}=C_{0}\left(1-\frac{2}{l} \sqrt{\frac{D t}{\pi}}\right)
$$

can be used for the calculation of diffusion coefficient at the lower value of $\sqrt{D t / l}$ than $0.5_{0}$ and the equation

$$
C_{a}=\frac{8 C_{0}}{\pi^{2}} e^{-\frac{D_{\pi} 2 t}{4 l 2}}
$$

\section{APPENDIX 1}

\section{Derivation of the alternative form of eq. (1)}

In the case of the steady flow of radioactive isotope in the capillary of length $l$ whose one open end is kept at zero concentration of radioactive isotope and the other closed end is kept no flow of radioactive isotope, the differential equation becomes

$$
\begin{aligned}
& C_{t}(x, t)=D C_{x x}(x, t) \quad(0<x<l, t>0) \\
& C(x, 0)=C_{0} \\
& C(0, t)=0, \quad C_{x}(l, t)=0 .
\end{aligned}
$$

When we apply the Laplace transformation to the above equations, we obtain

$$
\begin{aligned}
& p \bar{C}(x, p)-C_{0}=D \bar{C}_{x x}(x, p) \\
& \bar{C}(0, p)=0, \quad \overline{C_{x}}(l, p)=0
\end{aligned}
$$

The general solution of the above equation is

$$
\bar{C}(x, p)=\frac{C_{0}}{p}+A \cosh (l-x) q
$$

where

$$
A=-\frac{C_{0}}{p \cosh l g}, \quad q=\sqrt{\frac{p}{D}} .
$$

Now let us write the equation for $\overline{C_{a}}$ in the form

$$
\begin{aligned}
\bar{C}_{a}= & \frac{1}{l} \int_{0}^{l} \bar{C} d x=\left[\frac{C_{0}}{p l} x\right]_{0}^{l}+\left[\frac{C_{0} \sinh (l-x) q}{p l q \cosh l q}\right]_{0}^{l} \\
= & \frac{C_{0}}{p}-\frac{C_{0} \sinh l q^{*}}{p l q \cosh l q}=\frac{C_{0}}{p} \\
& -\frac{C_{0}}{p l q}\left(1+2 \sum_{n=0}^{\infty}(-1)^{n} e^{-2^{n l q}}\right) .
\end{aligned}
$$

Due to the inverse transformation eq. (C) becomes the result already quoted in this paper.

$$
\begin{aligned}
C_{a}= & C_{0}-\frac{2 C_{0}}{l} \sqrt{\frac{D t}{\pi}}-\frac{4 C_{0}}{l} \sqrt{\frac{D t}{\pi}} \sum_{n=1}^{\infty}(-1)^{n} i \\
& \quad \operatorname{erfc} \frac{n l}{\sqrt{D t}}
\end{aligned}
$$

can also be valid at the higher value of $\sqrt{D t} / l$ than $0.4_{2}$.

The calculated value of self-diffusion coefficient of silver ion in pure molten sodium nitrate at $350^{\circ} \mathrm{C}$ from the experimental result supports the justification of the above selection of an equation. The obtained diffusion coefficient of silver ion agrees well with that of chronopotentiometric and polarographic method.

\section{Acknowledgment}

The authors are indebted to Mr.T.Sugaya who performed the derivation of some equations.

$$
\begin{aligned}
= & C_{0}\left(1-\frac{2}{l} \sqrt{\frac{D t}{\pi}}\left(1+2 \sqrt{\pi} \sum_{n=1}^{\infty}(-1)^{n} i\right.\right. \\
& \left.\left.\operatorname{erfc} \frac{n l}{\sqrt{D t}}\right)\right)
\end{aligned}
$$

\section{APPENDIX 2}

Verification of identity of eq. $(3)$ with eq. (1)

We write for shortness

and therefore

$$
\sqrt{\lambda}=l q
$$

$$
p=\frac{\lambda}{l^{2}} D, \quad d p=\frac{D}{l^{2}} d \lambda
$$

From eq. (C) we can derive a following equation by the inversion theorem

$$
\begin{aligned}
& C_{a}= \frac{1}{2 \pi i} \int_{r-i \infty}^{r+i \infty} \frac{C_{0}}{p} e^{p t} d p-\frac{1}{2 \pi i} \\
& \int_{r-i \infty}^{r+i \infty} \frac{C_{0}}{p l q} \frac{\sinh l q}{\cosh l q} e^{p t} d p \\
&= \frac{1}{2 \pi i} \int_{r-i \infty}^{r+i \infty} \frac{C_{0}}{\lambda} e^{\frac{D}{l 2} \lambda t} d \lambda-\frac{1}{2 \pi i} \\
& \int_{r-i \infty}^{r+i \infty} \frac{C_{0}}{\lambda^{3 / 2}} \frac{\sinh \sqrt{\lambda}}{\cosh \sqrt{\lambda}} e^{\frac{D}{l 2} \lambda t} d \lambda,
\end{aligned}
$$

$$
\begin{aligned}
* \quad \frac{-C_{0} \sinh l q}{p l q \cosh l q}=\frac{-C_{0}}{p l q} \frac{e^{l q}-e^{-l q}}{e^{l q}+e^{-l q}}=\frac{-C_{0}}{p l q} \frac{e^{l q}-e^{-l q}}{e^{l q}\left(1+e^{-2 l q}\right)} \\
=\frac{-C_{0}}{p l q}\left(1-e^{-2 l q}\right) \sum_{n=0}^{\infty}(-1)^{n} e^{-2 n l q} \\
=\frac{-C_{0}}{p l q}\left(1-e^{-2 l q}\right)\left(1+\sum_{n=1}^{\infty}(-1)^{n} e^{-2 n l q}\right) \\
=\frac{-C_{0}}{p l q}\left(1-e^{-2 l q}+\sum_{n=1}^{\infty}(-1)^{n} e^{-2 n l q}-e^{-2 l q} \sum_{n=1}^{\infty}(-1)^{n} e^{-2 n l q}\right) \\
=\frac{-C_{0}}{p l q}\left(1-e^{-2 l q}+\left((-1)^{-2 l q}+(-1)^{2} e^{-4 l q}+(-1)^{3} e^{-6 l q}+\cdots\right)\right. \\
\left.\quad+\left((-1)^{2} e^{-2 l q-2 l q}+(-1)^{3} e^{-2 l q-4 l q}+\cdots\right)\right) \\
=\frac{-C_{0}}{p l q}\left(1+2 \sum_{n=1}^{\infty}(-1)^{n} e^{-n 2 l q}\right)
\end{aligned}
$$


where $r$ is to be so large that all the singularities of $\bar{C}_{a}$ lie on the left of the line $(r-i \infty, r+i \infty)$. The pole of the first term of right side of eq. (D) is $\lambda=0$. The residue at $\lambda=0$ is

$$
\lim _{\lambda \rightarrow 0}(\lambda-0) \frac{C_{0}}{\lambda} e^{\frac{D}{l^{2}} \lambda t}=C_{0} .
$$

As for the second term on the right side of eq. (D), the poles of this equation are $\lambda=0$ and $\lambda=-\pi^{2}\left(n+\frac{1}{2}\right)^{2}$. The residue at $\lambda=0$ is

$$
\lim _{\lambda \rightarrow 0}(\lambda-0) \frac{C_{0} \sinh \sqrt{\lambda}}{\lambda^{3 / 2} \cosh \sqrt{\lambda}} e^{\frac{D}{l 2} t}=C_{0}
$$

and the residue at $\lambda=-\pi^{2}\left(n+\frac{1}{2}\right)^{2}$ is

$$
\lim _{\lambda \rightarrow \alpha_{m}}\left(\lambda-\lambda_{m}\right) \frac{C_{0} \sinh \sqrt{\lambda}}{\lambda^{3 / 2} \cosh \sqrt{\lambda}} e^{\frac{D}{12} \lambda t}
$$

$$
\begin{aligned}
& =C_{0} \lim _{\lambda \rightarrow \lambda_{m}} \frac{\lambda-\lambda_{m}}{\sqrt{\lambda} \cosh \sqrt{\lambda}} \cdot \frac{\sinh \sqrt{\lambda}}{\lambda} e^{\frac{D}{12} \lambda t} \\
& =C_{0} \lim _{\lambda \rightarrow \lambda_{m}} \frac{1}{\frac{1}{2 \sqrt{\lambda}} \cosh \sqrt{\lambda}+\frac{1}{2} \sinh \sqrt{\lambda}} \text {. } \\
& \frac{(-i) \sin \left(-\pi\left(n+\frac{1}{2}\right)\right)}{-\pi^{2}\left(n+\frac{1}{2}\right)^{2}} e^{-\frac{D(2 n+1) 2 \pi^{2 t}}{4 l^{2}}} \\
& =\frac{-8}{\pi^{2}} C_{0} \frac{1}{(2 n+1)^{2}} e^{-\frac{D(2 n+1) 2 \pi 2 t}{4 l 2}} \text {, }
\end{aligned}
$$

where $\lambda_{m}$ is the value of the $m$ th $\lambda$. For all values of $n$ we obtain

$$
C_{a}=\frac{8 C_{0}}{\pi^{2}} \sum_{n=0}^{\infty} \frac{1}{(2 n+1)^{2}} e^{-\frac{D(2 n+1) 22^{2 t}}{4 l^{2}}} .
$$

